\title{
Morphogenèse et fabrication d'une forme structurellement informée : expériences pédagogiques sur les systèmes funiculaires
}

\author{
Roberta Zarcone ${ }^{1,2, *}$, Ahmed Hussein ${ }^{2,3}$, et Mohammad Mansouri ${ }^{1,2}$ \\ ${ }^{1}$ Ecole Nationale Supérieure d'Architecture et Paysage de Lille \\ ${ }^{2}$ Laboratoire GSA, Géométrie-Structure-Architecture, Ecole Nationale Supérieure d'Architecture \\ Paris-Malaquais \\ ${ }^{3}$ Ecole Nationale Supérieure d'Architecture Paris-Malaquais
}

\begin{abstract}
Résumé. Ce papier s'interroge sur la forme comme résultat d'un système qui interprète les enjeux contextuels, programmatiques et structuraux afin de concevoir et fabriquer des systèmes funiculaires à l'aide de méthodes à la fois physiques et numériques pour profiter de leurs potentialités structurales et géométriques. Les systèmes funiculaires sont des méthodes historiques de construction, mais les récentes méthodes numériques donnent de nouvelles possibilités architecturales et structurales à ce type de structures. Les expériences pédagogiques menées visent à trouver des formes (form finding) de haute qualité morphologique et de haute performance structurale. Ce papier présente la méthode pédagogique et, pour des cas concrets, l'algorithme de génération de la surface courbe pavée, en proposant différentes morphologies en réponses aux problématiques d'ordres géométriques et techniques, et la fabrication numérique à échelle un.
\end{abstract}

Mots-clés. Systèmes funiculaires, Modélisation paramétrique, Fabrication numérique

\begin{abstract}
Through physical and digital methods of form finding, this paper investigates the generation of funicular forms based on contextual, functional and structural considerations. Catenary structure systems are historical methods of construction, however, the advent of digital tools opens up new structural and architectural possibilities for its applications. This paper introduces an educational experience that applies the physical and digital methods of funicular form-finding to generate structures with high morphological quality and structural performance. Through a number of case studies, this paper introduces the applied educational method from the algorithm of form generation and panelling up to the digital fabrication of $1: 1$ models.
\end{abstract}

Keywords. Funicular systems, Parametric design, Computational fabrication.

\footnotetext{
*Corresponding author: zarconeroberta@gmail.com
} 


\section{Introduction}

Les vingt dernières années ont été caractérisées par un changement numérique en architecture (Carpo, 2013) qui a profondément influencé une discipline qui tentait de renouveler ses codes et son langage. Cette liberté formelle dans le processus de conception numérique s'est traduite en une grande variété de méthodes de construction et de processus de fabrication qui ont permis à ces réalités virtuelles de devenir des réalités construites.

La génération de la forme funiculaire nécessite un processus de conception qu'on pourrait définir comme structurellement informé reliant l'intention architecturale à la nécessité structurelle. L'un des premiers architectes à utiliser une telle approche fut Antonì Gaudi. Il a utilisé les méthodes de la statique graphique, les modèles suspendus et les maquettes en plâtre des structures caténaires pour trouver la forme la plus optimisée par rapport aux forces en jeu.

La méthode des maquettes physiques permet non seulement de contrôler une forme funiculaire en raison du fait que sa stabilité est principalement un problème d'équilibre statique et non de contraintes, mais elle représente surtout un outil de recherche morphologique (Rippmann et Block, 2013).

Basée sur les études de Culmann (1880) et Varignon (1725), la statique graphique est une méthode graphique pour construire la forme d'une corde inélastique suspendue en introduisant le polygone funiculaire et le polygone des forces. L'aspect purement graphique de cette méthode se révèle très utile pour des architectes éventuellement non formés sur des sujets mathématiquement plus complexes.

Les méthodes numériques développées ces dernières années, fournissent un environnement dynamique pour simuler le comportement des processus structuraux de recherche de forme. Récemment, un certain nombre d'outils numériques de form finding ont été développés, y compris CADenary et Kangaroo. Il existe deux méthodes principales de calcul numérique de simulation; la méthode de relaxation dynamique et la méthode de densité de force. Le plugin Kangaroo, utilisé dans ce travail, est basé sur la méthode de relaxation dynamique qui simule le comportement physique des câbles caténaires à travers une série de ressorts simples, de masses et de forces (Kilian et Ochsendorf, 2005).

Le développement de ces nouveaux outils a ouvert à la possibilité d'interopérabilité entre recherche morphologique des formes courbes et d'analyse structurelle, grâce à un flux d'informations continues dès les premières phases de conception (Elshafei et al., 2016). Cependant, il est important que les concepteurs sans connaissances approfondies des structures funiculaires soient capables d'interpréter correctement le processus de recherche de forme et ses résultats. L'interaction entre réel et virtuel, la frontière entre le monde réel et l'imaginaire peut apparaître floue. Nous visons à comprendre jusqu'où l'architecte reste acteur d'une conception architecturale structurellement informée. Les questions que nous posons ici sont alors : quelle est l'origine de la forme informatisée ? Et de quelle manière cette forme parvient-elle à exprimer les intentions et les contraintes architecturales et structurelles du projet?

\section{Form finding: approche numérique, approche physique et statique graphique}

Dans le but d'investiguer la tension entre immersion dans une réalité virtuelle en référence aux outils numériques, et émersion réelle en référence aux outils géométriques et physiques, nous reportons dans ce paragraphe différentes expériences pédagogiques menées à l'Ecole Nationale supérieure d'Architecture et de Paysage de Lille et à l'Ecole Nationale supérieure d'Architecture Paris-Malaquais. 
Dans ces expériences pédagogiques, l'interaction entre approches paramétrique et physique invite les étudiants à énoncer et transcrire leurs intentions de projet en actions paramétriques afin de définir de possibles variantes morphologiques. L'exercice pédagogique se déroule en deux temps : dans un premier temps il s'agit de trouver la forme qui traduit le mieux les intentions morphologiques des étudiants par rapport au contexte, au programme, à la circulation et/ou au parcours solaire. Les inputs paramétriques sont d'ordre géométriques (points d'appuis, longueur de la corde, forme du maillage de base, hauteur) et d'ordre physique (création du modèle élastique du matériau et définition des forces appliquées). La recherche morphologique est réalisée avec une approche à la fois numérique (Kangaroo) et physique (maquettes d'étude). Une fois les variantes morphologiques définies (output: surface ou courbe funiculaire), la deuxième étape de conception prévoit la transformation de la forme dans une construction. Il s'agit donc de combiner des intentions architecturales (en lien par exemple avec des paramètres environnementaux tels que les flux lumineux par exemple) avec des contraintes d'ordre structurales (ouverture des joints ou glissement) et de faisabilité de fabrication. Ces contraintes/intentions doivent se traduire en opérations de transformations géométriques sur des paramètres (input) définis par l'étudiant afin d'obtenir des variantes de morphologies constructives (figure 1).

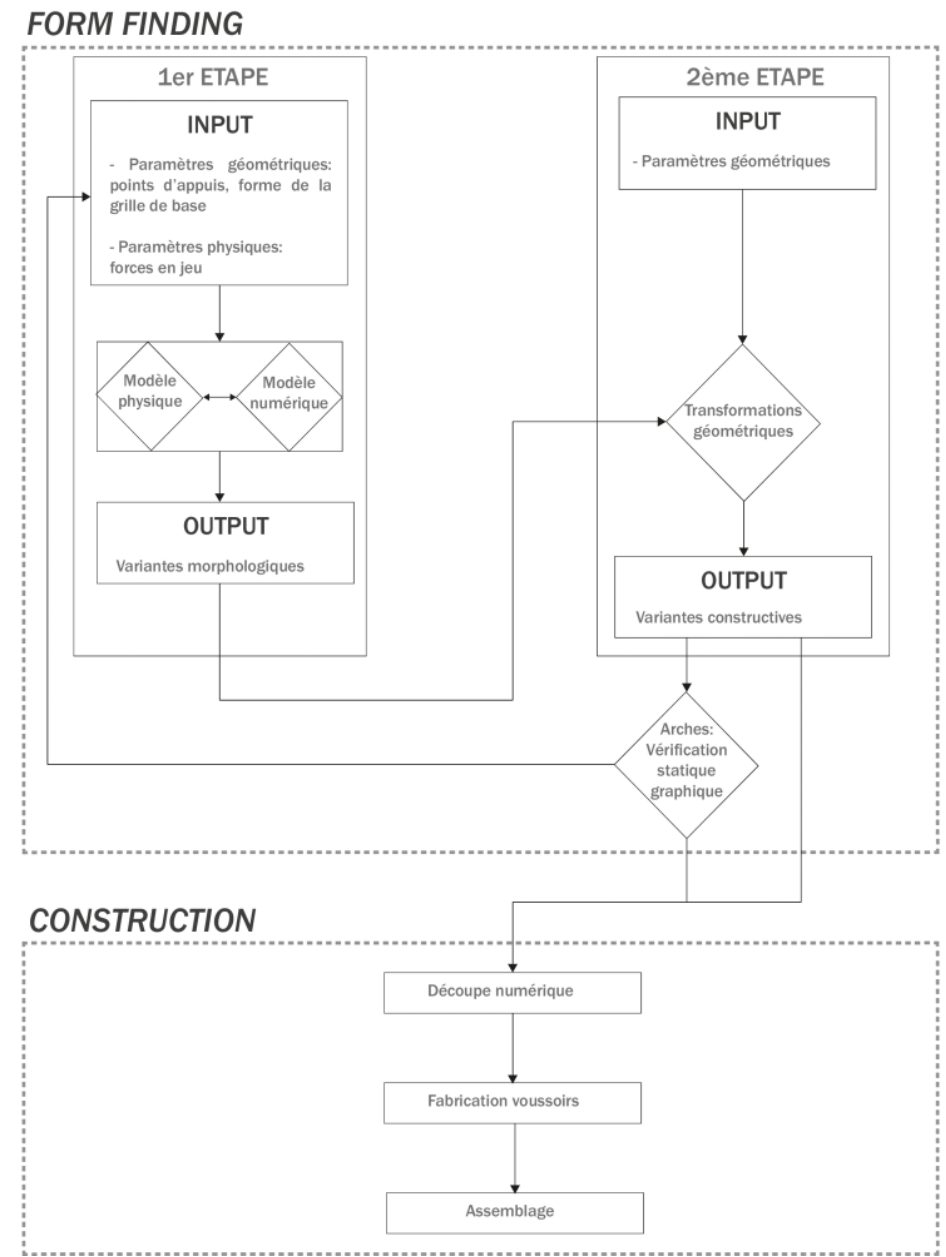

Figure 1. Organigramme résumant le processus de conception structurellement informé jusqu'à la fabrication. 


\subsection{L'exemple de l'arche}

Dans le cas des arches, nous nous sommes basés sur une approche de la forme comme un prototype constitué de voussoirs en pierre. Il a été question de réinterpréter la morphogenèse d'une arche maçonnée avec la triple approche (maquette physique, statique graphique, maquette numérique) afin de pousser à la limite les contraintes structurales de ce type de système. Les étudiants ont travaillé sur la conception d'une arche plane sur le site de la cafétéria de l'école de l'ENSAP de Lille. Dans ce travail, les étudiants organisés par groupe sont invités à communiquer le comportement physique des chaînes métalliques aux valeurs numériques des ressorts et des masses dans Kangaroo pour se rapprocher du comportement du matériau. La calibration du funiculaire entre la chaînette physique et la simulation numérique est basée sur le relevé des dimensions de la courbe caténaire fait sur la chaînette (portée entre les deux points d'accroche et flèche). Cette proportion de largeur et de hauteur est ensuite reproduite dans le modèle numérique par le réglage de la force de gravité dans Kangaroo pour atteindre les mêmes proportions. Les étudiants sont ainsi amenés à formuler des propositions différentes (figure 2).
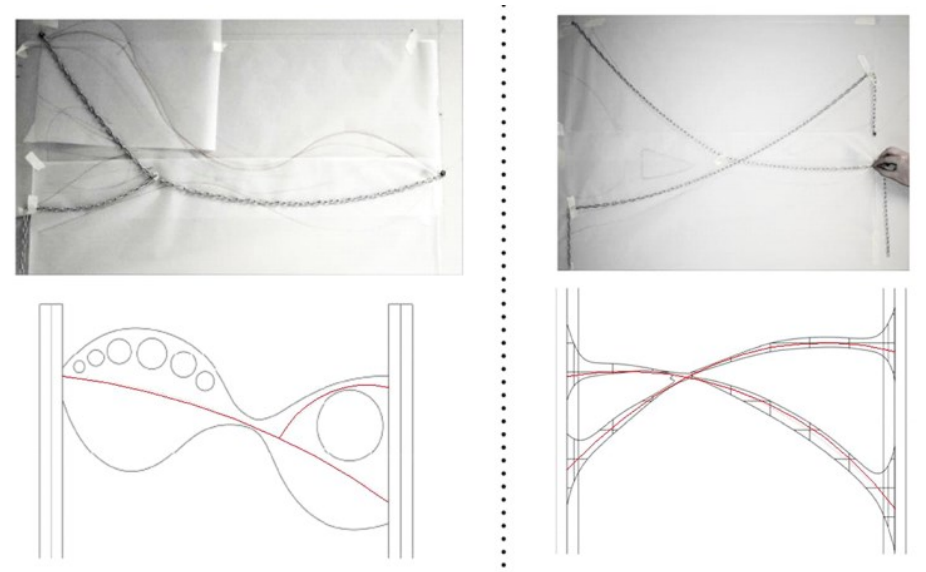

Figure 2. Deux propositions morphologiques à l'aide des chaînettes et de Kangaroo.

D'un point de vue structurel, la rupture d'une arche s'avère le plus fréquemment par des mécanismes qui correspondent à l'ouverture des joints ou à des glissements localisés sur ces joints. Pour éviter le premier mécanisme, nous faisons l'hypothèse d'une loi de contact simplement limitée à la traction et illimitée à la compression. Dans ce cas, si la résultante des pressions de contact est à l'intérieur de la section de contact, l'équilibre est possible. Les courbes d'intrados et d'extrados de l'arche représentent donc les limites de non résistance (Benvenuto, 2010). Une fois recherchée la courbe funiculaire à l'aide de ce jeu d'interaction entre modélisation numérique et modélisation physique, les étudiants conçoivent l'arche (avec son épaisseur) de manière à pousser à la limite la recherche morphologique toute en tenant en compte des limites de non résistance. Le traçage du calepinage vise à éviter les phénomènes de glissement de joints. On suppose que la façon optimale de charger les joints est obtenue lorsque la composante tangentielle de la réaction transmise par le joint est nulle. Pour ce faire, il faut que le joint soit le plus orthogonal à la courbe des pressions. Les étudiants sont donc menés à traduire ces contraintes structurales en transformations géométriques. Ils passent ensuite à la vérification à l'aide de la statique graphique (figure 3). 


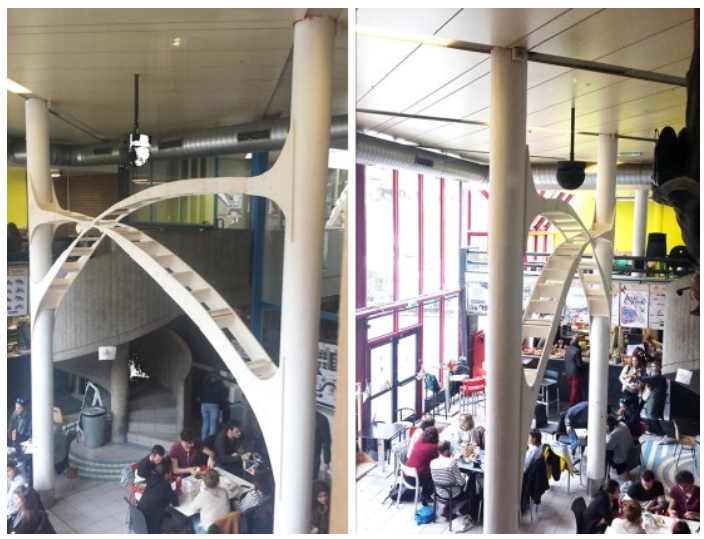

Figure 3. Prototype final réalisé.

Dans une autre expérience, les étudiants ont travaillé sur la conception d'une arche 3D lors de la semaine intensive numérique à l'ENSA Paris-Malaquais. Dans ce cadre, les étudiants avaient la contrainte de concevoir un espace défini par des arches avec trois points d'appuis à différentes hauteurs. Les arches devaient reposer les unes sur les autres. La vérification avec la statique graphique a été faite à l'aide du logiciel Geogebra. L'objectif n'était pas seulement de vérifier le funiculaire de chaque arche mais aussi de vérifier l'état d'équilibre des forces au niveau des trois nœuds du funiculaire, ce qui revient à dire vérifier l'équilibre d'un point dans l'espace (figure 4 et figure 5).
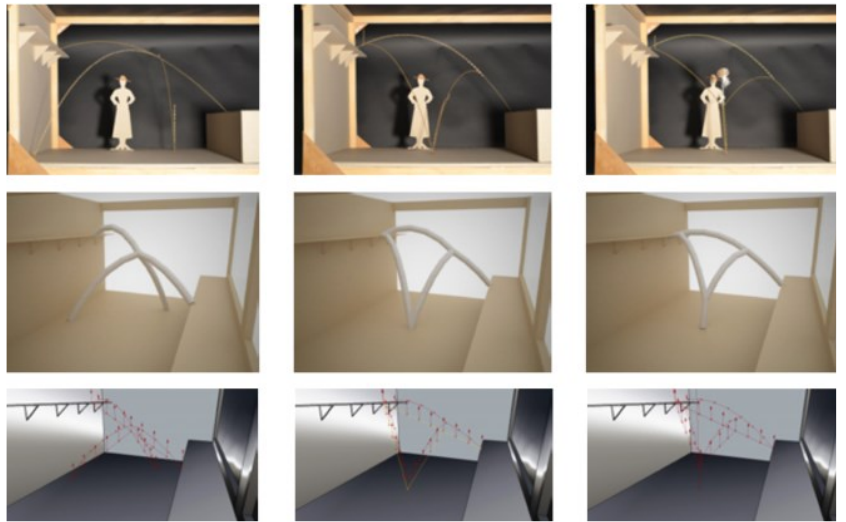

Figure 4. L'arche dans l'espace : Trois propositions morphologiques à l'aide des chaînettes et de Kangaroo.
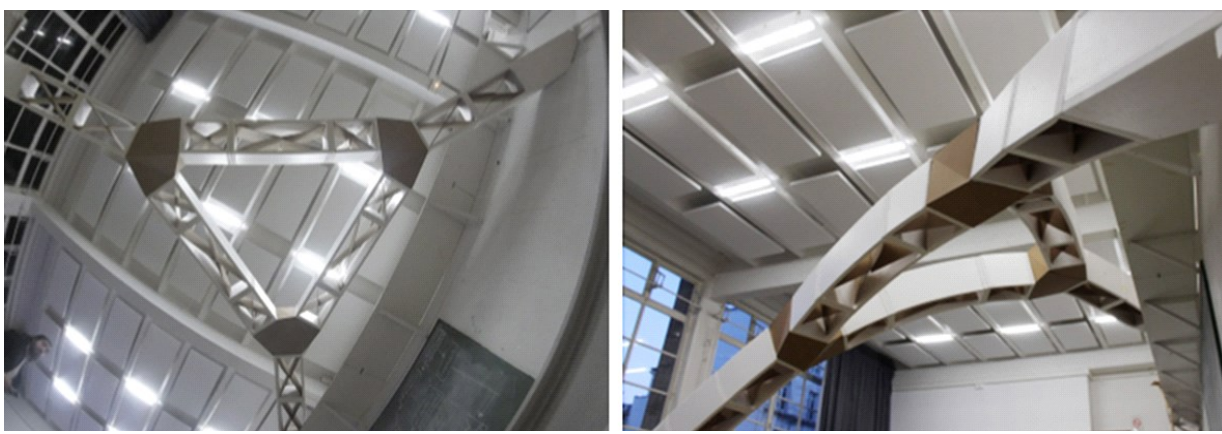

Figure 5. Prototype final. 


\subsection{L'exemple des surfaces funiculaires}

Dans le cas des surfaces funiculaires, la forme a été recherchée uniquement à l'aide du logiciel Kangaroo, avec plusieurs aller-retours entre réel et virtuel pour résoudre les problèmes, d'ordre géométriques et constructifs, dus à la forme et au matériau utilisés pour la fabrication.

Une première expérience de conception numérique de surfaces funiculaires a été faite avec des modèles en plâtre. Ces derniers sont basés sur la méthode The hanging cloth reversed introduite par Heinz Isler en 1959 (Chilton, 2012). Dans cette expérience, les étudiants ont mesuré et calibré les propriétés de courbure du tissu par rapport au modèle numérique afin de rapprocher la simulation numérique au comportement physique du tissu. Les relevés des quatre points d'accroche et de la hauteur de la surface du modèle physique sont reportés sur le modèle numérique en calibrant les valeurs des forces gravitationnelles. Le processus de fabrication est basé sur la coupe du tissu en fonction de la taille du modèle numérique, la suspension et la solidification avec le plâtre. L'avantage de cette technique de fabrication est qu'elle donne une surface caténaire solide et mince dont la courbure est très proche du modèle numérique. Le défaut est l'influence de la forme du bord sur la courbure de la surface qui crée des rides si elle n'est pas correctement développée à partir du modèle numérique (figure 6).
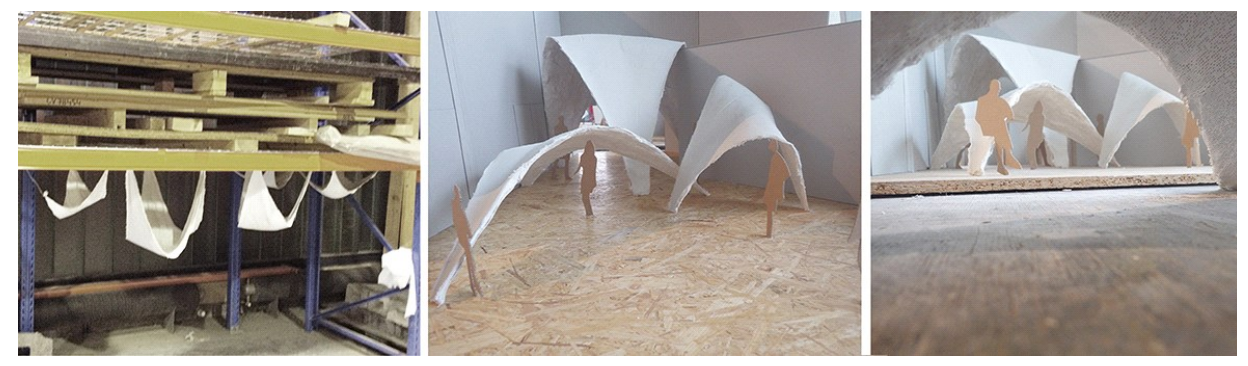

Figure 6. Modèles en plâtre

Après un travail de recherche de forme sous différentes contraintes réelles liées au site, le passage de la variante morphologique numérique à la construction nécessite des démarches expérimentales d'aller-retour entre la géométrie de la surface à double courbure, les matériaux (carton $2 \mathrm{~mm}$ ), l'échelle de la maquette, le principe d'assemblage et les méthodes de fabrication. Un des problèmes principaux lorsque nous travaillons avec des surfaces funiculaires est lié à la " développabilité » de la surface. Si d'une part le matériau avec lequel nous construisons les surfaces est le carton, de l'autre, les surfaces funiculaires générées sont souvent en doubles courbures. Pour résoudre ce problème, les étudiants doivent dans un premier temps procéder à la discrétisation de la surface.

Toutes ces questions peuvent être traitées par différentes approches qui donnent des possibilités constructives et morphologiques différentes. Nous allons par la suite présenter quelques variantes constructives conçues par les étudiants.

Dans une première expérience, la surface caténaire (double courbure) est discrétisée par un pavage triangulaire à l'aide du plugin Lunchbox (figure 7). Le pavage ouvre à des possibilités morphologiques, en permettant de générer des apports de lumière par des ouvertures au centre de chaque tuile. Les dimensions des perforations doivent prendre en considération à la fois la stabilité de la structure et l'intensité de l'éclairage. Les ouvertures des tuiles sont alors inversement proportionnelles à la hauteur des tuiles, plus les tuiles sont proches du sol, plus petite est la surface des perforations. Les contraintes dues au pavage sont liées à la recherche de la densité correcte (nombre et dimensions des tuiles). En effet une densité trop petite entraine une discrétisation de la surface qui détermine une 
approximation risquant de s'éloigner trop de la surface courbe souhaitée. Au contraire une densité trop grande détermine des contraintes de faisabilité constructive dues à de possibles imprécisions de montage par rapport aux surfaces de contact.

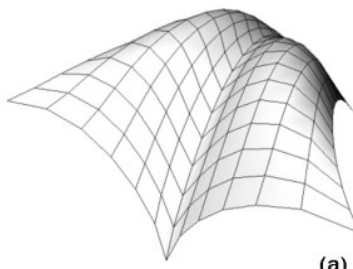

(a)

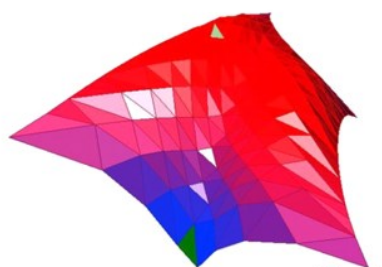

(b)

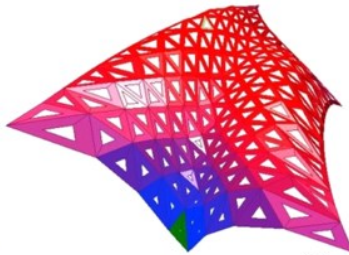

(c)

Figure 7. (a) Surface funiculaire double courbure, (b) Triangulation de la surface pour créer des tuiles planes, (c) Perforation des tuiles pour l'éclairage.

Une autre approche morphologique pour la fabrication d'une surface funiculaire pavée avec un pavage hexagonal ou quadrilatéral propose de construire des pyramides sur la base de chaque tuile de la surface. Le sommet de la pyramide se trouve sur le vecteur normal à la tuile partant du centre (figure 8). La distance entre le sommet de la pyramide par rapport au polygone de base donne une variété de morphologies différentes (figure 9). En effet, plus cette distance est petite plus la nouvelle géométrie épouse la surface originaire (figure 10).

Cette méthode permet d'obtenir des surfaces triangulaires (cotés latéraux de la pyramide) planes pour la fabrication.

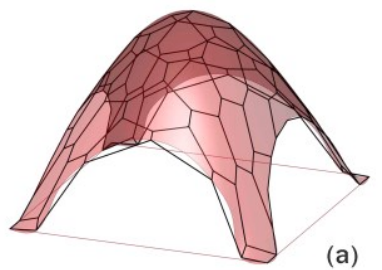

(a)

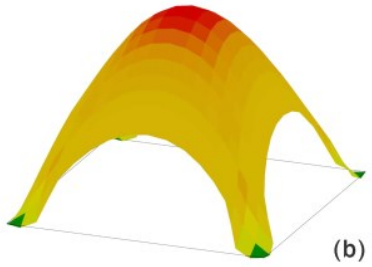

(b)

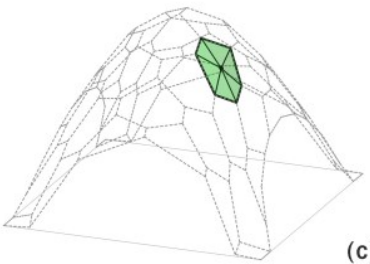

(c)

Figure 8. (a) Surface pavée avec pavage hexagonal, (b) Analyse de la courbure gaussienne, (c) Triangulation de l'hexagone.
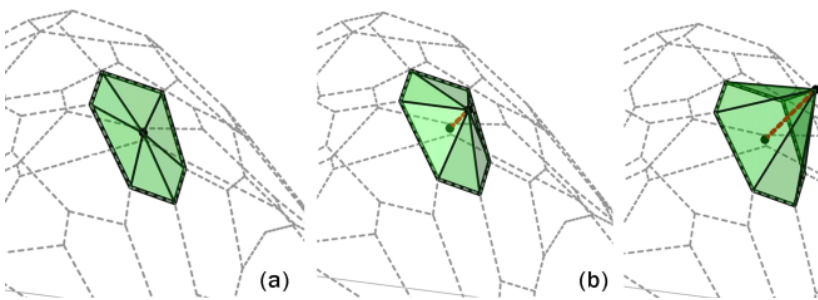

(c)

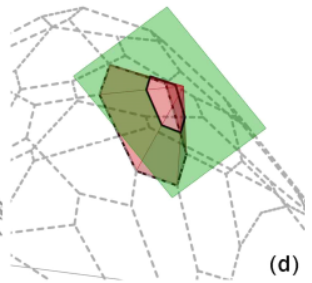

Figure 9. (a) Triangulation de l'hexagone, (b, c) Construction à différentes hauteurs, (d) Découpe de la pyramide. 


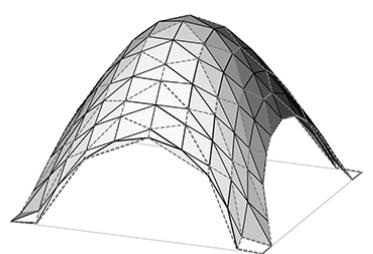

(a)

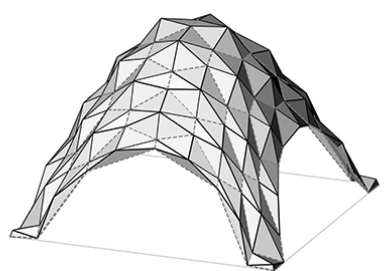

(b)

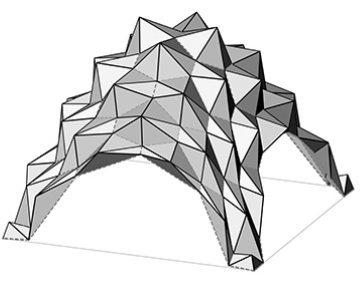

(c)

Figure 10. Variantes de morphologies constructives proposées.

Dans le but d'optimiser à la fois la distribution de matière et d'obtenir un jeu de lumière à l'intérieur de la voûte, les étudiants ont coupé les pyramides avec un plan orthogonal au même vecteur normal qui a permis la construction de la pyramide (figure 9). Le plan est paramétrable afin d'avoir des variations de dimensions des ouvertures. Le paramétrage de la taille des ouvertures peut être lié à un paramètre environnemental. Une solution pourrait être de paramétrer les ouvertures proportionnellement au flux lumineux passant à l'intérieur ou encore à la radiation solaire sur la surface (figure 11). Le thème de la matérialité est ainsi traité avec interaction entre la matière solide et celle invisible donnée par le jeu de lumière et d'ombres.
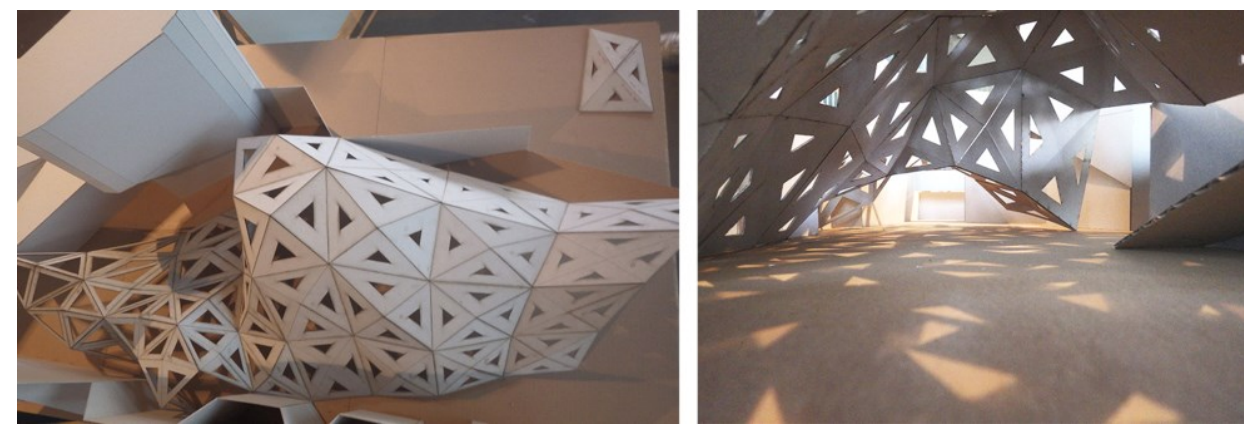

Figure 11. Maquette avec ouvertures paramétrées par rapport au parcours solaire. Jeu de lumière et d'ombres.

En ce qui concerne les assemblages des différentes tuiles entre elles, il est nécessaire de donner une épaisseur aux éléments afin de donner un blocage correct des voussoirs entre eux et d'éviter le phénomène de glissement. La solution la plus simple est d'extruder les bords des polygones pour trouver des faces. Cependant, l'orientation du vecteur normal de chaque sommet du pavage sur la surface est différente, donc l'extrusion des polygones du pavage suivant ces normales rend des faces en torsion. Afin d'éviter ce problème de nondéveloppabilité une possibilité est de faire des extrusions vers un point. Il s'agit de construire des pyramides sur la base de chaque polygone du pavage en ayant comme sommet en commun le centre de la sphère inscrite dans la surface (figure 12). Les étudiants ont par la suite paramétré ainsi le processus de découpage de ces pyramides par des plans afin d'avoir la largeur nécessaire des faces d'assemblage. De cette manière, les surfaces de contact seront alignées entre elles et le flux de forces se trouvera à l'intérieur de cette épaisseur. De plus, la tolérance donnée par la présence d'un joint (dans notre cas réalisé avec de la colle) permet d'assurer le blocage des voussoirs sans phénomènes de glissement. Pour toutes ces expériences, la préparation de la surface pour la fabrication numérique avec la découpe laser a été réalisée avec une numérotation de chaque tuile dans le modèle 3D (figure 13). Le processus d'assemblage respecte l'orientation et le numéro des tuiles (figures 14 et 15 ). 

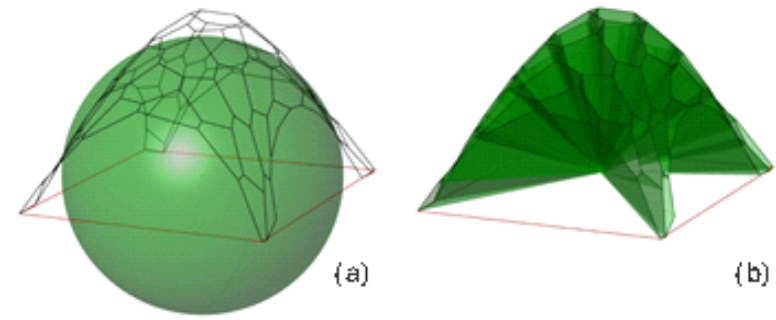

(b)

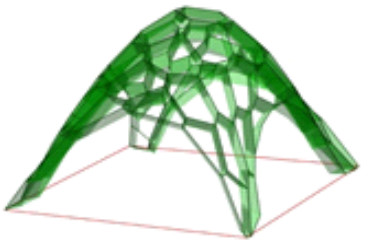

(c)

Figure 12. Construction géométrique pour obtenir les surfaces de contact. (a) Construction de la sphère inscrite dans la surface, (b) Traçage des droites et des surfaces entre les sommets des hexagones et le centre de la sphère, (c) Découpe avec des plans pour obtenir les épaisseurs.
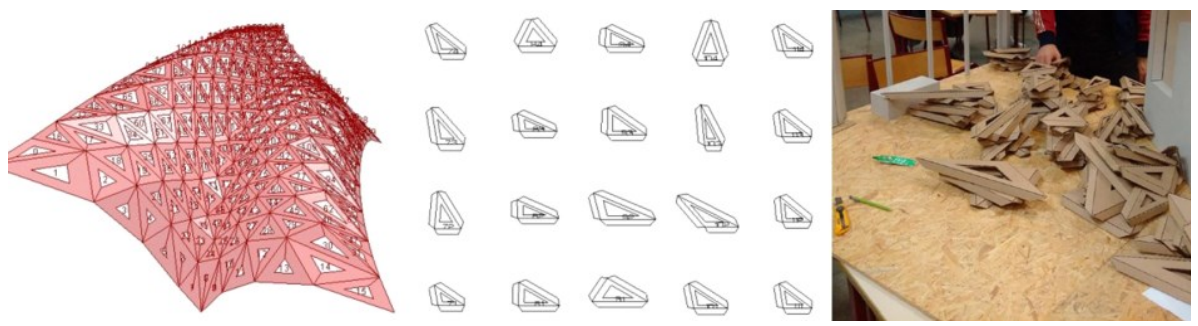

Figure 13. Numérotation des tuiles et développement pour le découpage laser.
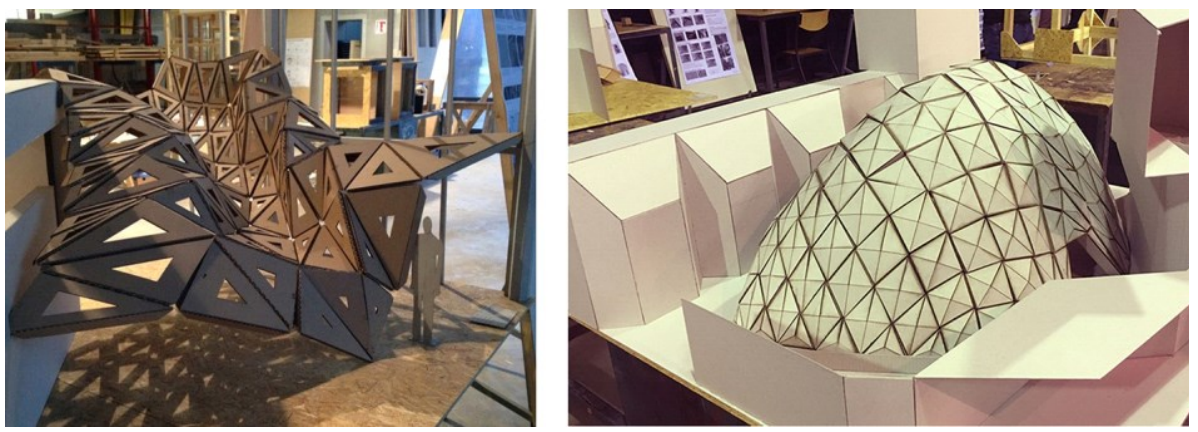

Figure 14. Maquettes construites (a) avec pavage triangulaire, (b) avec pavage quadrilatéral.
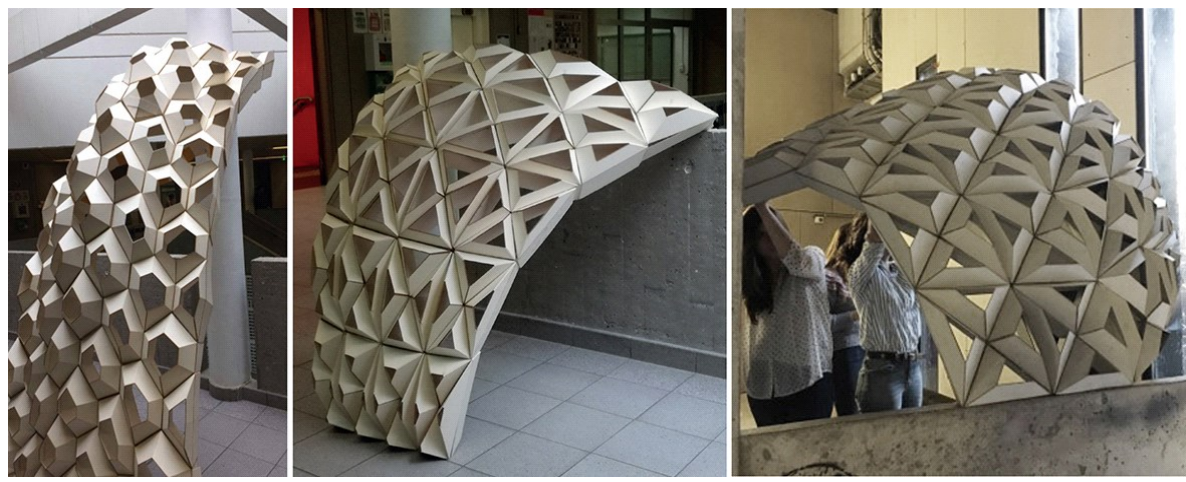

Figure 15. Prototypes réalisés avec la méthode des pyramides coupés. 


\section{Conclusion}

Ce papier s'interroge sur la forme comme résultat d'un système qui interprète les enjeux contextuels, programmatiques et structuraux afin de concevoir et de fabriquer des surfaces funiculaires. Les exercices pédagogiques visent à mener les étudiants à organiser une démarche de projet morphologique par le dessin, la statique graphique et la manipulation de maquettes, réelles et numériques.

La première expérience vise à construire une arche en poussant au maximum la recherche formelle, tout en intégrant les contraintes structurelles. Cela a permis d'initier les étudiants à la recherche de la forme dans un processus structurellement informé. La compréhension de la nécessité d'une approche intégrée pour la recherche morphologique représente un point de difficulté pour les étudiants. De ce fait, certains d'entre eux ont tendance à privilégier une approche (numérique ou physique) au détriment de l'autre. Les étudiants sont amenés à intégrer dans le processus de conception des contraintes non seulement d'ordre programmatique mais aussi d'ordre structurel, environnemental et géométrique. La difficulté de cet exercice réside dans un manque de compétence géométrique de base d'un grand nombre d'étudiants; la nécessité de traduire des contraintes différentes en transformations géométriques entraine des difficultés de compréhension et donc d'anticipation de possibles problèmes liés à la fabrication et à l'assemblage.

Dans l'ensemble des manipulations, cette pédagogie vise à mener les étudiants à s'interroger sur la recherche de l'équilibre optimal entre les intentions architecturales propres à l'activité de l'architecte et les contraintes d'un programme défini et d'un site réel dans une démarche de conception structurellement informée. Cette approche interactive entre recherche morphologique physique et modélisation à l'aide du logiciel permet aux étudiants de faire plusieurs aller-retours entre le réel et le virtuel, en mettant en place un flux d'informations continu visant à l'optimisation dans un contexte à plusieurs contraintes.

\section{Bibliographie}

Benvenuto, E. (2010). La scienza delle costruzioni e il suo sviluppo storico. Rome: Storia e Letteratura.

Carpo, M. (2013). The digital turn in architecture 1992-2012. Hoboken: John Wiley \& Sons.

Chilton, J. (2012). Form-Finding and Fabric Forming In the Work of Heinz Isler. In J. Orr, M. Evernden, A. Darby \& T. Ibell (Eds.), ICFF2012: Proceedings of Second International Conference on Flexible Formwork (pp.84-91). Bath: BRE CICM.

Cullman, C. (1880). Traité de statique graphique. Paris : Dunod éditeur.

Elshafei, A., Hussein, A., Mansouri, M., Vekinis, M., Zarcone, R., \& Brocato, M. (2016). Couverture d'une surface d'égale pente par un pavage de Penrose: conception numérique et fabrication. In J.-P. Goulette \& B. Ferries (Eds.), SCAN'16 Toulouse (pp.59-70). Nancy : Presses universitaires de Nancy.

Kilian, A., \& Ochsendorf, J. (2005). Particle-Spring Systems for Structural Form Finding. In C. Gantes (Ed.), Journal of the International Association for Shell and Spatial Structures, 46, 77-84.

Rippmann, M., \& Block P. (2013). Funicular Funnel Shells. In C. Gengnagel, A. Kilian, J. Nembrini, F. Scheurer (Eds.), Rethinking prototype. Proceedings of the Design Modelling Symposium. Berlin, 2013 (pp. 75-89). Berlin : Springer Edition.

Varignon, P. (1725). Nouvelle mécanique ou statique. Tome 1. Paris : C. Jombert. 